\title{
ANTONI DE MARTÍ I FRANQUÈS, LA GENERACIÓN ESPONTÁNEA Y LA TRANSFORMACIÓN DE LOS ORGANISMOS ${ }^{1}$
}

\author{
Agustí Camós \\ CHIC-UAB e IS Miquel Martí i Pol \\ agusti.camos@gmail.com
}

Recibido: 14 septiembre 2012; Aceptado: 3 abril 2013.

Cómo citar este artículo/Citation: Camós, Agustí (2013), “Antoni de Martí i Franquès, la generación espontánea y la transformación de los organismos", Asclepio 65 (2): p022. doi: http://dx.doi.org/10.3989/asclepio.2013.22

RESUMEN: Antoni de Martí i Franquès fue un naturalista y científico catalán nacido en 1750 y muerto en 1832, cuyos trabajos más conocidos han sido sus estudios sobre la composición del aire y sobre la reproducción de los vegetales, donde tuvo un cierto reconocimiento internacional. Sin embargo, buena parte de su trabajo experimental que no publicó, lo dedicó al estudio de la fisiología vegetal y de la generación espontánea. Estaba seguro que era capaz de producir experimentalmente organismos vegetales muy simples a partir de restos de seres vivos, agua y luz. Analizaremos los datos que disponemos sobre estos trabajos experimentales, sus resultados, el contexto en que se realizaron y las posibles influencias. También estudiaremos sus ideas acerca de la transformación de los organismos, la influencia de Lamarck, así como los planteamientos que compartía con la teoría evolucionista del naturalista francés.

PALABRAS CLAVE: Generación espontánea; siglo XVIII y XIX; Evolución; Cataluña; España.

ANTONI DE MARTÍ I FRANQUÈS, SPONTANEOUS GENERATION AND ORGANISM'S TRANSFORMATION

ABSTRACT: Antoni Martí Franquès was a naturalist and scientist born in Catalonia, Spain (1750- 1832). His main contributions were on the vegetal reproduction as well as on the composition of the air, from which he achieved a significant international recognition. However, most of his unpublished experimental works were done on vegetal physiology and the spontaneous generation. He was convinced that he could produce very simple vegetable organisms from the organic material, water and light. Here, we discuss the few data that we know about his experimental works, the results, the context and the external influences. Also, we discuss his concepts about the transformation of the organisms, the influence of Lamarck, and the ideas on evolution that Marti shared with the French naturalist.

KEY WORDS: Spontaneous generation; XVIII and XIX century; Evolution; Catalonia; Spain.

Copyright: (c) 2013 CSIC. Este es un artículo de acceso abierto distribuido bajo los términos de la licencia Creative Commons Attribution-Non Commercial (by-nc) Spain 3.0. 


\section{INTRODUCCIÓN}

Antoni de Martí i Franquès fue un personaje un tanto singular en la España de la segunda mitad del siglo XVIII y primeros decenios del siglo XIX, pero similar al de otros de ricos propietarios agrícolas y científicos amateurs que vivían contemporáneamente en diversos países europeos. Tenemos constancia de su importante trabajo experimental, pero debido a su personalidad y a las dificultades de su entorno solamente publicó un trabajo científico. Escribió al menos cinco memorias de las cuales conocemos dos leídas en la Real Academia de Ciencias Naturales y Artes de Barcelona (RACAB), y una leída en la Real Academia Médico Práctica de Barcelona (RAMPB); solo conocemos el título de las otras dos (Quintana, 1935, p. 216).

La memoria que tuvo mayor repercusión fue la que llevaba por título "Sobre la cantidad de aire vital que se halla en el aire atmosférico", y fue leída en la RACAB en 1790. En ella Martí establecía los porcentajes de oxígeno y de nitrógeno que contenía el aire atmosférico, así como la constancia de dichos porcentajes al medir la composición del aire en diversas condiciones. El trabajo experimental de Martí mejoraba los cálculos anteriores realizados por Priestley y Lavoisier entre otros grandes científicos. Extractos de dicha memoria se publicarían posteriormente en Madrid, Londres y Berlín, y tendría resonancia en medios científicos europeos y norteamericanos. Sin embargo, a menudo al citarla se modificó el nombre de su autor, llamándole De Marty o Macarty, dificultando por ello el conocimiento del verdadero origen de dicha memoria (Grau, 2011, pp. 34-40)

Otra memoria que tuvo una cierta repercusión fue la que leyó en la RAMPB con el título de "Sobre los sexos y fecundación de las plantas", donde contradecía algunos de los trabajos de Spallanzani sobre la reproducción de los vegetales. El científico italiano afirmaba que había comprobado experimentalmente que algunas plantas como el cáñamo, la espinaca o la sandía, podían reproducirse a partir de la parte femenina de la flor sin la intervención del polen. Sin embargo Martí demostró experimentalmente que esto no era posible. Esta memoria fue publicada y tuvo repercusión en algunos medios; apareció un resumen de la misma en el Diario de los nuevos descubrimientos de todas las ciencias físicas publicado en Madrid en $1793^{2}$, y también se cita dicho trabajo en el Seminario de Agricultura y Artes dirigido a los párrocos ${ }^{3}$, publicado en la misma ciudad.

Sin embargo, a través del material manuscrito de Martí que se conserva así como por diferentes referencias de amigos y contemporáneos suyos, conocemos que hizo un enorme trabajo experimental dedicado al estudio de la fisiología y a la generación de los vegetales, temas sobre los que no llegó a publicar nada. En este artículo trataremos de situar sus trabajos experimentales sobre la generación espontánea, el contexto en que se realizaron, las influencias que recibió, y sus ideas sobre la transformación de los organismos.

\section{LA VIDA DE ANTONI DE MARTÍ}

El estudio más completo sobre Martí lo publicó en 1935 uno de los pioneros de la historia de la ciencia en Cataluña y en España, Antoni Quintana Marí. En los últimos decenios se han aportado a este trabajo de referencia algunos interesantes estudios parciales (Rovira, 1982; Quintana, 1985 y 1992; Grapí, 2001; Grau, 2011; Bernat, 2011, entre otros).

Martí nació en el año 1750 en Altafulla, una población costera situada unos pocos kilómetros al norte de la ciudad de Tarragona, en el seno de una familia de la nobleza con muchas tierras e intereses en la incipiente industria del textil, del algodón y de la alfarería. Buena parte de su tiempo lo destinó a la gestión de su patrimonio y a la importación y exportación de bienes.

Su formación como científico y naturalista fue básicamente autodidacta. Fue miembro de la RACAB y de la RAMPB, y entre 1800 y 1801 realizó un largo viaje por Europa visitando diversas instituciones científicas en París, Londres, La Haya, Ámsterdam y Bruselas. Disponía de una amplia biblioteca que estaba al día de las publicaciones europeas y mantuvo contactos con diversos científicos, particularmente franceses. También disponía de un buen laboratorio donde hacía estudios químicos sobre los gases, así como otros trabajos experimentales relacionados con la fisiología y la generación de los vegetales. Además había constituido un notable herbario así como una apreciable colección de minerales y rocas.

Prueba de su enorme trabajo experimental sobre fisiología vegetal y la generación espontánea, son las aproximadamente tres mil páginas manuscritas que se conservan en la Biblioteca-Hemeroteca de Tarragona. En estos manuscritos se recogen únicamente los trabajos experimentales que realizó entre 1816 y 1828 , siendo ya muy mayor puesto que tenía entre 66 y 78 años, pero sorprendentemente contiene decenas de millares de observaciones. No se lee con facilidad por su estado de conservación y por la multitud de abreviaturas que contiene, y tan solo ha sido trascrito en una pequeña parte (Quintana, 1935, pp. 210-237). Sin embargo, a partir de estos datos podemos hacernos una idea de la gran cantidad de material manuscrito sobre sus investigaciones que habría acumulado a lo largo de toda su vida. La causa fundamental de la pérdida de la mayor parte de estos documentos fue la destrucción realizada por las tropas de Napoleón cuando entraron en la ciudad de Tarragona tras su si- 
tio en 1811. Las tropas francesas saquearon y destruyeron el laboratorio que Martí tenía instalado en su casa, desapareciendo la mayor parte de sus trabajos realizados hasta la fecha. En este momento Martí ya tenía 61 años, por lo que desapareció el testimonio del trabajo experimental de la mayor parte de su vida.

\section{EL PROBLEMA DE LA GENERACIÓN ESPONTÁNEA}

Como ya henos dicho, uno de los temas sobre los cuales más trabajo experimental realizó Martí y que le causó grandes preocupaciones fue la generación espontánea, es decir, la posibilidad de que se pudiera generar materia viva a partir de materia inanimada sin la intervención de ningún ser vivo. Se trata de uno de los problemas biológicos más debatidos desde el mundo clásico hasta la actualidad, que ha ocupado la atención de muchos pensadores en diferentes países y en diferentes épocas, adhiriéndose a un amplio espectro de puntos de vista filosóficos y teológicos (Farley, 1977, p. 1). Curiosamente, su derrocamiento como paradigma ha sido celebrado al menos tres veces: una vez en el siglo diecisiete, otra en el dieciocho y otra en el diecinueve (Mendelsohn, 1976, p. 40). Sin embargo existe una cierta confusión en relación a lo que significa la generación espontánea. En realidad bajo estas palabras se incluyen tres procesos diferentes.

Por un lado está lo que se denomina la generación equívoca, que consiste en que una especie nacería a partir de otra diferente; fue universalmente rechazada en el siglo XVIII (Mclaughlin, 2005, p. 80). Por lo que conocemos, Martí nunca se refirió abiertamente a este tipo de generación espontánea, aunque como veremos, algunos de sus planteamientos parecen cercanos al transformismo que no se aleja mucho de esta concepción.

Por otro lado tenemos la heterogénesis, la posibilidad de que se produzcan seres vivos a partir de restos de organismos. Como veremos, Martí defendió la existencia de este proceso, puesto que estaba convencido que lo había comprobado experimentalmente en numerosas ocasiones.

Por último tenemos la abiogénesis, la formación de seres vivos a partir únicamente de materia inorgánica, que como también veremos, Martí admitía como una posibilidad. La abiogénesis fue ampliamente aceptada durante buena parte del siglo XIX por muchos evolucionistas a pesar de los experimentos de Pasteur, puesto que permitía dar una explicación materialista del origen de la vida; el mismo Darwin tenía una posición favorable en relación a la posible existencia de este proceso aunque nunca la defendió de forma pública (Peretó, 2009, pp. 403-405.)

\section{LAS POLÉMICAS ACERCA DE LA GENERACIÓN ESPON- TÁNEA EN VIDA DE MARTÍ}

La generación espontánea fue defendida por muchos filósofos en la Grecia clásica, como Anaximandro, Anaxímenes o Demócrito. Pero fue la defensa que de ella hizo Aristóteles, la que causó mayor impacto en el mundo occidental a causa de la enorme influencia del gran filósofo griego.

En los primeros años del siglo XVII no había muchas razones para dudar de la existencia de la generación espontánea. Sin embargo, a finales de este siglo la explicación cartesiana de la generación espontánea ya parecía insuficiente, sobre todo después de descubrir la complejidad que tenían los organismos vivos cuando se observaban a través del microscopio (Farley, 1977, pp. 9-10).

En la segunda parte del siglo XVII diversos autores, como Francesco Redi, William Harvey o John Ray, desarrollaron una fuerte ofensiva contra la generación espontánea condicionados por sus creencias religiosas, pero apoyándose en notables trabajos de observación y experimentación (Mendelsohn, 1976, p. 48). En estos años, el intenso debate sobre este tipo de generación se mezcló con las investigaciones sobre la reproducción sexual, la búsqueda de los óvulos y el papel de estos en el proceso reproductivo.

A finales del siglo XVII había un amplio consenso alrededor de la negación de la generación espontánea. En su lugar se defendía la concepción según la cual los organismos estaban ya preformados en su germen originario, preformacionismo, y que este organismo preformado en el caso de los animales se encontraba en el óvulo, ovismo. No obstante, algunos hechos continuaron siendo difíciles de explicar como el origen de los gusanos parásitos y el de los organismos monstruosos, y a ellos se unieron nuevas observaciones tales como el descubrimiento de los espermatozoides, los fenómenos de regeneración de algunos organismos como la hidra, o la hibridación vegetal.

A principios del siglo XVIII el triunfo del newtonianismo volvió a favorecer la generación espontánea y la epigénesis, teoría alternativa al preformacionismo según la cual el desarrollo embrionario se produciría a partir de una masa inicial homogénea que se iría diferenciando. En este contexto, algunos prestigiosos naturalistas que disfrutaban de una considerable influencia como Buffon, defendieron la generación espontánea haciendo que de nuevo esta tuviera una gran aceptación entre los estudiosos de la naturaleza.

Según Buffon, cuando ocurría la descomposición de la materia viva, las moléculas orgánicas ya no se encontraban adheridas a la estructura de las diferentes partes del organismo y por ello se liberaban. Esta concepción proporcionaba las bases para la aceptación 
de una forma de generación espontánea, la heterogénesis. Los trabajos experimentales que había desarrollado le habían convencido de que se podrían haber originado tantos seres vivos a partir de la mezcla de moléculas orgánicas, como a través de los procesos de descendencia (Farley, 1977, pp. 23-24). Sin embargo, en la situación en la que se encontraría la naturaleza en nuestro tiempo la propensión a producir materia viva que tendría formaría seres vivos muy sencillos, puesto que la mayor parte de esa materia orgánica estaría ya integrada en los cuerpos de otros vivientes (Caponi, 2009, p. 694).

Otro personaje clave en la defensa de la generación espontánea en estos años fue el sacerdote católico inglés John Tuberville Needham. Se trata de un naturalista que intentó conciliar la religión y la ciencia, en particular con los datos que aportaba la experimentación y el razonamiento. Cristiano ferviente y católico convencido, pero tolerante, intentó persuadir a los cristianos de que antes de condenar las nuevas ideas que aportaban los naturalistas intentaran comprenderlas (Roger, 1993, p. 511). Como Buffon, rechazaba el ovismo y apoyaba la epigénesis, y a menudo su pensamiento se confundió con el del naturalista francés sin que él hiciera nada para evitarlo, y por ello fue acusado de haber dado argumentos a los ateos. Estos planteamientos lo condenaron a la soledad y a la incomprensión, cuando su objetivo era defender un modelo de generación espontánea que evitara el mecanicismo y el materialismo (Roe, 1983, p. 160).

Needham era un experto microscopista que hizo un gran trabajo experimental. En el año 1748 trabajó unos meses en París con Buffon, realizando una serie de experimentos y observaciones según los cuales los dos naturalistas creían haber confirmado experimentalmente la generación espontánea. Para Needham la generación de nuevos organismos no podía producirse al azar, sino únicamente en aquellas circunstancias que Dios había fijado, y a través de las leyes que había establecido. Además, tenía que existir una fuerza vegetativa que necesitaba la presencia del aire, que hacía posible la generación de nuevos seres vivos.

El principal oponente de la generación espontánea en estos años, y por tanto oponente de Buffon y Needham, fue el también sacerdote católico Lazzaro Spallanzani. También fue un hábil experimentador que repitió los trabajos de Needham, pero tomando una serie de precauciones para evitar la aparición de gérmenes procedentes del aire, comprobando que de esta forma no se producía el fenómeno de la generación espontánea. La disputa entre los sacerdotes católicos fue larga y enconada. Para Needham, las manipulaciones de Spallanzani destruían el espíritu vital del aire impidiendo la generación, mientras que para este último la aparición de microorganismos era causada por la entrada de gérmenes procedentes del aire. A pesar de su enfrentamiento científico, mantuvieron una larga correspondencia y unas buenas relaciones (Roger, 1993, p. 725).

En la lucha en contra de la generación espontánea Spallanzani no estaba solo. Junto al italiano se encontraban otros prestigiosos naturalistas como René Réaumur, Charles Bonnet y Albrecht von Haller, todos pertenecientes a la misma familia intelectual y espiritual. Defendían los derechos de la experiencia, y realmente eran muy buenos observadores, pero ante todo eran mecanicistas y cristianos ortodoxos (Roger, 1993, p. 731).

Las tres primeras décadas del siglo XIX fueron testimonio del nuevo triunfo de la generación espontánea. Los naturalistas más destacados defendían especialmente esta generación en el caso de los infusorios y de los gusanos parásitos, basándose en experiencias empíricas (Farley, 1977, p. 31). Entre estos naturalistas se encontraba Jean-Baptiste Lamarck.

\section{MARTÍ Y LA GENERACIÓN ESPONTÁNEA}

Como ya hemos visto, Martí no publicó nada acerca de la generación espontánea, pero a partir de diferentes testimonios de sus contemporáneos y los registros manuscritos de su trabajo experimental, sabemos que fue un tema sobre el que hizo numerosos experimentos y reflexiones. A través de uno de sus amigos y confidentes, el clérigo Fèlix Torres Amat, sabemos que estaba convencido de que había conseguido generar formas vegetales simples a partir de materia inerte, por tanto que sabía producir de forma experimental la generación espontánea. Escribía Torres Amat en su obra dedicada a los escritores catalanes al referirse a los trabajos experimentales de Martí:

Estos ensayos tuvieron por objeto la producción artificial de los vegetales por la organización de la materia inorgánica; habiendo alcanzado a formar a su arbitrio varias confervas, tremolas y otras plantas celulares, teniéndose presentido haberse extendido igualmente a algunas vasculares (1836, p. 381).

Otro testimonio de los trabajos de Martí sobre la generación espontánea fue el que hizo otro de sus amigos, el médico Jaume Parcet. Dicho médico también era socio de la RAMPB, y envió a esta institución el 20 de noviembre de 1832 una necrología en recuerdo de su amigo que había muerto dos meses antes. Esta necrología es de gran interés ya que su autor explica que frecuentó durante muchos años el gabinete laboratorio de Martí y que mantuvo con él largas conversaciones (Parcet, 1907, p. 700). En la explicación que hace Parcet del trabajo de Martí en el gabinete quedan claramente reflejados los experimentos sobre la generación espontánea: 
Este gabinete, despreciable en apariencia e inestimable por su esencia es donde con su sublime penetración podemos decir que ha sorprendido a la Naturaleza, pues que facilitando combinaciones de principios ha conseguido organizaciones vegetales, las cuales estaba cierto que no procedían de otro símil. Se le ha oído repetidas veces que a un cadáver lo reduciría todo a una masa térrea, y que ésta la dispondría después de un modo que todos sus principios, o la mayor parte de ellos, constituirían organizaciones vegetales improcedentes también de símil; y pertenecientes estas y las anteriores a la última clase de Ligneo (sic) (Parcet, 1907, p. 697).

Un tercer testimonio contemporáneo lo encontramos a través de Josep Arrau, que fue un importante pintor y académico de la RACAB. Arrau junto con otros jóvenes constituyó en Barcelona en los años 1829 y 1830 una notable tertulia en la que también participaba Martí, cuando el naturalista tarragonés ya tenía cerca de ochenta años. Otros participantes en dicha tertulia eran Carles Martí, sobrino del naturalista tarragonés, Joan Agell, que sería unos años más tarde académico de la RACAB y catedrático de química de la Universidad de Barcelona, y Marià de la Pau Graells, quien se convertiría en un gran entomólogo y catedrático de la Universidad de Madrid. En la descripción de la tertulia, Arrau se refiere a las explicaciones de Martí sobre sus trabajos relativos a la generación espontánea:

Entre estas discusiones se logró varias veces que el señor Martí esplanase los medios que se valía para lograr artificialmente la formación y crecimiento de las plantas acuáticas criptógamas que el denominaba trémulas y más corrientemente materia verde, y la teoría que en su concepto podía admitirse para explicar ese maravilloso fenómeno (Elias de Molins, 1895, vol. II, pp. 93-94).

Tal y como recoge Arrau y podemos comprobar a través de los manuscritos de Martí, el término que utilizaba para referirse a los pequeños organismos que habría formado por generación espontánea era «materia verde». Se trata de un término común en Europa en estos años, alrededor del cual se desarrolló un notable debate del que trataremos más adelante (Bory de Saint Vincent, 1823, pp. 324-336).

Por último nos referiremos al testimonio más importante, a las palabras del propio Martí en los interesantísimos manuscritos que se conservan de sus investigaciones y reflexiones, que como hemos dicho, solo recogen sus últimos doce años de trabajos experimentales. Así, en los resultados de 1816 podemos leer en su apartado 5:

Un vaso con un excremento de palomo, y poca agua no dio gas ni mata. [materia] verde en Dbre. [diciembre] hasta el cabo de 11 días; y un frasquito tapado de medida lleno de la agua del vaso en que estuvo el excto. [excremento] por espacio de 24 horas dio gas y mata. verde al cabo de 5 días. Dbre. 23 y 27 exp.

[experimento] 3 y 7 (Quintana, 1935, p. 229).

Aquí Martí deja constancia de creer haber producido organismos vegetales simples, materia verde, a partir de agua y excremento de palomo. Además describe que cambiando las condiciones en que se desarrollaba el experimento, el proceso podía producirse de forma más o menos rápida. En el resultado 13 del mismo año describe un proceso parecido a partir de estiércol y agua:

La mata. [materia] verde en invierno suele comunte. [comunmente] manifestarse abtos. [abiertos] vasos con agua y estiércol, después de 8 a 12 días. Dbre. [Diciembre] 27 y 31, exp. [experimento] 7 y 15 (Quintana, 1935, p. 230).

Como podemos comprobar Martí estaba convencido de que podía producir organismos vivos a partir de agua y restos de materia orgánica, es decir, creía haber comprobado experimentalmente la heterogénesis. Pero también se preguntaba si era posible producir organismos vivos a partir de la materia inerte que no procedía de ningún ser vivo, es decir producir procesos de abiogénesis. Esto es lo que escribía en el manuscrito titulado "Problemas y resultados" en el apartado número 15:

Así como el gas carbo. [carbónico] se descompone por la mata. [materia] verde, apropiándose esta su carbón con que se alimenta, y crece, y soltando oxigeno en gas, dicho gas carbónico con la agua pura y sin intermedio alguno, formaría mat. verde? (Quintana, 1935, p. 215).

\section{POSIBLES INFLUENCIAS}

Sabemos que Martí estaba al día de los debates científicos que se estaban produciendo en Europa a través de las publicaciones periódicas que recibía y de la amplia biblioteca que poseía. Por esta razón creemos que conocía las polémicas contemporáneas sobre la generación espontánea, así como las que se habían producido en otras épocas, especialmente las desarrolladas en los siglos XVII y XVIII. Tenemos la suerte de que se ha conservado parte de su biblioteca, pero sabemos que había sido todavía bastante más amplia. Por una parte ya nos hemos referido al asalto que sufrió su casa y su gabinete por parte de las tropas napoleónicas, con la consiguiente destrucción de libros, manuscritos y material de investigación. Pero además tenemos constancia de que parte de la biblioteca que se había conservado tras su muerte, sufrió considerables pérdidas en años posteriores.

Conocemos este dato a través de un descendiente suyo, el naturalista, ingeniero de montes y propietario agrario Joaquim Castellarnau. Según su testimonio, que recoge Quintana, de niño jugaba en la biblioteca de Martí que había heredado el tío de Castellarnau, 
Gaietà Martí Veciana, que a su vez era nieto del naturalista tarragonés (Quintana, 1935, pp. 196-197). Cuando ya era mayor Castellarnau hacía las siguientes reflexiones:

Muchos años más tarde contemplé con profunda pena, que las estanterías de la vetusta biblioteca estaban casi vacías. ¿Donde habían ido a parar los libros que con tanto goce había hojeado en mi juventud, y que despertaron en mi quizás mi inclinación a los estudios, que más tarde han ocupado la mayor parte de mi vida? (Quintana, 1935, p. 87) ${ }^{4}$.

Profundizando un poco más se refiere en primer lugar a la desaparición de libros de gran valor, como Herbarum vivae eicones de Otto Brunfells o la Historia stirpium de Leonard Fuchs. No da ninguna explicación a esta desaparición, pero no sería aventurado aducir motivos económicos. Después Castellarnau se refiere a una posible razón de la expurgación de la biblioteca de Martí, aludiendo al "alma excesivamente religiosa» de alguno de sus descendientes que condenaba la generación espontánea porque estaba en contra de la ortodoxia católica. Así pues, parece que alguno de los descendientes de Martí hizo desaparecer la mayor parte de los libros que trataban sobre la generación espontánea, y por tanto que los pocos libros que se conservan donde se trata este tema podrían ser tan solo una pequeña parte de los que había poseído. No obstante, de entre lo que queda de la biblioteca, que a pesar de las pérdidas a las que nos hemos referido es notable, hemos podido encontrar algunos libros que nos pueden orientar en relación a las influencias que sobre este tema pudo haber recibido el naturalista tarragonés.

Como hemos visto, uno de sus grandes defensores de la generación espontánea durante el siglo XVIII fue Buffon. De entre los libros que se han conservado de la biblioteca de Martí, encontramos siete volúmenes del naturalista francés que corresponden a diversas colecciones; el tomo XI de la Histoire naturelle generale et particuliere, los tomos I, III y IV de la Histoire naturelle des oisseaux, el tomo $\mathrm{VI}$ de la Histoire naturelle des Mineraux, y los tomos I y II de la segunda edición les Les Époques de la nature (Quintana, 1935, p. 275). Se trata pues de tomos sueltos de diversas colecciones que muy probablemente el acaudalado Martí tendría completas, puesto que Buffon era uno de los naturalistas más prestigiosos de su época.

En los libros que se conservan se encuentra alguna referencia a la generación espontánea, aunque no totalmente explícita. Por ejemplo, en el volumen primero de Les Époques de la Nature en la tercera época de la historia de la Tierra, podemos leer una referencia al origen de los vegetales por generación espontánea aunque sin usar estos términos:

Las moléculas orgánicas vivas han existido desde que los elementos de un calor dulce han podido incorpo- rarse con sustancias que componen los cuerpos organizados; ellas han producido sobre las partes elevadas del globo una infinidad de vegetales (Buffon, 1778, p. $115)^{5}$.

No se conserva en lo que queda de la biblioteca de Martí la obra en que Buffon puso de manifiesto más claramente su posición con respecto a la generación espontánea. Se trata del segundo tomo de la Histoire naturelle, générale et particulière, avec la description du cabinet du roy, publicado en 1749 . Es muy probable que esta obra hubiese formado parte de la biblioteca de Martí puesto que desarrollaba uno de los problemas biológicos que más le preocuparon. Pero precisamente por hacer una clara defensa de la generación espontánea, esta obra podría haber sido una de las víctimas del «alma excesivamente religiosa» de alguno de los descendientes de Martí a que se refería Castellarnau.

Tampoco aparece en lo que se conserva de su biblioteca ninguna obra de Needham, quien junto a Buffon fue el gran defensor de la generación espontánea en la segunda parte del siglo XVIII. Siendo Martí un católico convencido que quería hacer compatibles sus convicciones religiosas con sus pensamientos científicos, Needham podía haber sido uno de los autores que más podrían haberle interesado por su condición de sacerdote católico que también quería hacer compatible la generación espontánea con sus creencias, y que también aceptaba una interpretación no literal del Génesis. Las obras de Needham también pudieron ser víctimas de la citada "alma excesivamente religiosa».

En cambio, sí que se conserva de la biblioteca de Martí una de las obras del gran adversario de Needham, el también sacerdote católico Spallanzani. Se trata de Éxperiences pour servir a l'histoire de la generation des animaux et des plantes (Quintana, 1935, p. 284), la obra en que Spallanzani afirmaba que determinados vegetales podían reproducirse a partir únicamente de la parte femenina de las flores sin la intervención del polen, en consonancia con sus planteamientos preformacionistas y ovistas.

Martí tuvo un enfrentamiento intelectual y experimental con el sacerdote italiano, puesto que en su memoria "Sobre los sexos y fecundación de las plantas" rebatía experimentalmente que determinadas plantas superiores pudieran reproducirse únicamente a partir de la parte femenina de la flor, poniendo de manifiesto diversos errores cometidos por Spallanzani en el desarrollo de sus experimentos (Bernat, 2011). Por tanto, Martí había de confiar poco en los trabajos experimentales de Spallanzani con los que habría tratado de desmentir los resultados de Buffon y sobre todo los de Needham, puesto que había comprobado personalmente que el sacerdote italiano cometía importantes errores en este tipo de trabajos. En conse- 
cuencia es muy posible que también se sintiera desde el punto de vista experimental más cerca del trabajo del sacerdote católico inglés.

La obra de Spallanzani que se conserva en la biblioteca de Martí es la traducción al francés del original italiano, realizada por el naturalista suizo y pastor protestante Jean Senebier, que además incluye un largo escrito de este último. Este naturalista ginebrés trabajó experimentalmente sobre la «materia verde» y sobre otro de los temas que preocuparon a Martí, la nutrición de las plantas. Los trabajos de Senebier pudieron tener una notable influencia en Martí, puesto que se conservan tres obras en su biblioteca relacionadas con el estudio de la influencia de la luz solar sobre los vegetales, además de los cinco volúmenes de su fisiología vegetal (Quintana, 1935, p. 127).

El texto de Senebier que se incluye en la traducción de la obra de Spallanzani consta de 88 páginas donde el naturalista suizo hace un esbozo de la historia de los organismos. Defiende el preformacionismo afirmando que la generación no es una creación sino el desarrollo de un ser preexistente, y que negar este hecho nos acercaría al ateismo (Senebier, 1787, pp. XI-XIII). Más adelante al afirmar que absolutamente todos los organismos, incluso los infusorios, han estado preformados, niega indirectamente la posibilidad de la generación espontánea:

No hay en absoluto hombres, animales, plantas, animáculos de infusión que no hayan existido, diría casi vivido desde hace seis mil años, y que desde este tiempo, no hayan experimentado un desarrollo sucesivo en el seno de las hembras donde estaban depositados (Senebier, 1787, p. XXIX).

A continuación Senebier trata de desacreditar la generación espontánea aún sin citarla, asociándola al azar, la ignorancia, y también al ateismo:

... los fetos no existen desde toda la eternidad, puesto que son finitos y sucesivos, estos no son la obra del azar, que no es más que una palabra, o el recurso de la ignorancia, o el asilo del ateismo que no ha reflexionado, puesto que estos fetos son muy complejos ...

Martí, que como veremos compartía con Senebier muchas de sus ideas sobre la denominada «materia verde», no podía participar de su postura con respecto a la generación de los organismos. Pero aunque sus presupuestos religiosos no coincidieran totalmente, es probable que las reflexiones que hacía en relación a la vinculación del ateísmo con la aceptación de la generación espontánea preocuparan a Martí puesto que era un ferviente cristiano católico.

El texto de Senebier acaba con una defensa de los experimentos que Spallanzani presenta en la obra sobre la reproducción de los vegetales, que Martí impugnaría en la memoria a la que ya nos hemos re- ferido. A continuación aparece la refutación de unos escritos del fisiólogo vegetal Jan Ingen-Housz, y finalmente se encuentra el núcleo central de libro, la traducción de la obra de Spallanzani sobre la generación de animales y plantas. A lo largo de toda ella defiende el preformacionismo ovista, apoyándose en su trabajo experimental y en el de otros notables naturalistas suizos como Bonnet y Haller, y ataca los planteamientos de Buffon, en especial la epigénesis. Pero en este texto de Spallanzani no aparece ninguna referencia directa a la generación espontánea.

Otro naturalista que pudo haber influenciado a Martí fue Lamarck, un autor que aceptó la generación espontánea muy tardíamente, cuando ya tenía cerca de sesenta años, para convertirla en un elemento fundamental de su teoría evolucionista. En lo que se conserva de la biblioteca de Martí encontramos una obra de Lamarck, el Système des Animaux sans vertèbres publicada en París en el año 1801. Se trata de una destacada obra del naturalista francés puesto que en ella aparece la primera formulación escrita de su teoría evolucionista. Esta primera formulación aparece en la reproducción del discurso de apertura del año 1800 de los cursos de zoología que impartía en el Muséum national d'Histoire naturelle de París, que se incluye al principio de la obra.

Lamarck tardó mucho en aceptar la generación espontánea debido a que una de las grandes convicciones que tenía sobre la naturaleza era que existía un "hiatus inmenso" (Lamarck, 1801, p. 5) entre la materia bruta y la viva, haciendo que el paso directo de la primera a la segunda fuera enormemente difícil, en realidad imposible para él durante la mayor parte de su vida. Esto le supuso un problema importante en el desarrollo de su modelo evolucionista partiendo de su concepción materialista, puesto que le dificultaba comprender cómo se originaban los seres vivos.

Parece que fue aceptando la generación espontánea a partir de sus estudios sobre los infusorios, considerando que esta forma de vida animal tan elemental sí que podía producirse a través de dicho proceso (Corsi, 2001, p. 90). A partir de 1802 Lamarck aceptó totalmente la generación espontánea de las formas vivas más sencillas, convirtiéndose en un paso crucial para el desarrollo de su teoría evolucionista (Burkhardt, 1977, p. 139). Sin embargo la obra de Lamarck que se conserva de la biblioteca de Martí fue escrita entre 1800 y 1801, cuando Lamarck empezaba a aceptar el proceso, aunque solo se atrevió a defenderlo veladamente.

En el citado discurso de apertura Lamarck hace alguna vaga alusión a la posibilidad de la generación espontánea, pero sin utilizar en ningún caso la expresión. Ocurre por ejemplo cuando Lamarck se refiere a que es la naturaleza quien produce los animales más simples: «Esta facilidad, esta abundancia, esta rapidez 
con la cual la naturaleza produce, multiplica y propaga los animales más simplemente organizados» (Lamarck, 1801, p. 21).

También al tratar en el mismo discurso sobre los pólipos, el grupo en el cual Lamarck incluía todavía a los infusorios en estos años, vuelve a hacer afirmaciones solo comprensibles en el contexto de la generación espontánea:

Ellos constituyen el último de los escalones que se pueden señalar en este interesante reino, y es entre ellos donde se encuentra el término desconocido de la escala animal, en una palabra los esbozos de la animalización que la naturaleza forma y multiplica con tanta facilidad en las circunstancias favorables (Lamarck, 1801, p. 41).

Ya en el propio texto zoológico del Système des Animaux sans vertèbres escrito poco después del discurso, encontramos algunas referencias a la generación espontánea todavía más claras, pero sin llegar a usar estos términos u otros parecidos. Al referirse de nuevo a los infusorios escribe:

Tenemos razones para creer que para los seres vivos más sencillos, el calor, que es la madre de las generaciones y una especie de alma de los seres vivos, opera concurrentemente con la humedad, que es necesaria para efectuar cualquier desarrollo orgánico; esta disposición y estado de las partes crean la vida, ya sea vegetal, ya sea animal, en las pequeñas masas gelatinosas aglomeradas que la naturaleza forma con tanta facilidad en las circunstancia favorables (Lamarck, 1801, pp. 391-392).

En este último texto Lamarck escribe de nuevo cómo la naturaleza crea por sí misma los pequeños infusorios. En obras posteriores, singularmente en la Philosophie zoologique publicada en 1809 y la Histoire naturelle des animaux sans vertèbres publicada entre 1815 y 1822, Lamarck fue desarrollando su modelo evolucionista y defiendió de forma clara y contundente la generación espontánea. No podemos descartar que al menos alguna de las obras citadas se encontrara en la biblioteca de Martí, pero que aquella «alma excesivamente religiosa» también las hubieran hecho desaparecer. En este sentido hay que recordar que Lamarck durante buena parte del siglo XIX fue un autor a quien se realacionó con la revolución francesa, el materialismo y el ateísmo, como puede comprobarse en los ataques que sufrió en el Estado Español por parte de autores como Josep Planellas o Josep Letamendi (Camós, 2007a, p. XLII). Por tanto sus obras también pudieron haberse convertido en un objetivo de aquellos que expurgaron la biblioteca de Martí por motivos religiosos.

Hemos de añadir a las obras citadas el Journal de physique, de chimie, d'histoire naturelle, del cual se conserva solo el tomo de 1803 en la biblioteca de
Martí, pero que posiblemente había de contener más volúmenes de esta publicación periódica puesto que fue en ella donde apareció uno de los extractos de la memoria de Martí sobre el aire atmosférico. La editaba Jean-Claude Delamétherie, y sabemos que en ella se informaba de los debates que se estaban produciendo en Europa en torno a la generación espontánea (Corsi, 2012, p. 18).

Así pues, entre las obras que se conservan de la biblioteca de Martí encontramos algunas donde aparece reflejada la defensa que hacían algunos autores de la generación espontánea, como Buffon y Lamarck, mientras también encontramos autores que la atacaban, como Senebier. Pero es bantante probable que en la biblioteca también hubieran figurado otras obras de estos y otros autores como Needham y Spallanzani, donde la defensa o la descalificación de la generación espontánea era más explícita.

Por otra parte, en los últimos decenios del siglo XVIII y en los primeros decenios del XIX se desarrolló en Europa un notable debate entorno a la denominada "materia verde», la sustancia que se generaba cuando los rayos solares incidían sobre el agua. Los científicos no estaban de acuerdo en la naturaleza de dicha materia, y sorprendentemente vincularon poco este debate con el de la generación espontánea. Como hemos visto Martí hizo diversos experimentos sobre la producción de dicha «materia verde».

El primero que describió el proceso y que le dio el nombre de materia verde a la sustancia que se producía fue Joseph Priestley (Schofield, 2004, p. 139 y 154; Bory de Saint-Vicent, 1823, p. 325). Inicialmente Priestley describió el material formado como un sedimento mucoso de color verde, pero sin atribuirle características vivas; sin embargo más adelante la caracterizó como sustancia vegetal afirmando que se trataba de Confervas, pero continuó negando que se tratara de un caso de generación espontánea basándose en argumentos teológicos (Farley, 1977, p. 45). Martí, que también denominaba Confervas a los organismos vegetales producidos, tenía en su biblioteca diversos volúmenes de la obras de Priestley (Quintana, 1935, p. 281), por lo que tenía que conocer sus experiencias que posiblemente fueron una de sus fuentes de inspiración.

Otro científico que participó en la polémica y del que ya hemos hablado fue Senebier. Repitió los experimentos de Priestley, y de acuerdo con él creyó que la materia verde era de carácter vegetal aunque de una especie distinta de Conferva. Por otra parte según Senebier esta materia verde con el tiempo se iba desarrollando hasta formar un vegetal suficientemente grande para ser visible a varios pasos de distancia (Bory de Saint-Vicent, 1823, pp. 328-329). Estas descripciones de Senebier concuerdan bastante con los 
datos que conocemos de las experiencias de Martí. De todos modos Senebier, como Priestley, también negaba la existencia de la generación espontánea.

También se refiere a esta polémica Ingen-Housz en el segundo volumen de su libro Expériences sur les végétaux que se conserva de la biblioteca de Martí. En ella Ingen-Houz se refería a los experimentos de Priestley (134), pero estaba en desacuerdo con que la materia verde fuera vegetal. Defendía que era materia animal formada por multitud de insectos verdes. Martí nunca defendió esta opinión.

\section{LA PROXIMIDAD DE LAS IDEAS DE MARTÍ A PLANEA- MIENTOS EVOLUCIONISTAS CONTEMPORÁNEOS}

Como hemos dicho, la generación espontánea era una pieza clave en el modelo evolucionista de Lamarck, y Martí conocía esta teoría por lo menos a partir de la obra del naturalista francés que sabemos que tenía en su biblioteca. Además, no es aventurado pensar que incluso pudiera haber llegado a conocer en París al naturalista francés en el viaje que realizó por Europa, visitando universidades, academias e institutos. Sus biógrafos afirman que «le proporcionó la amistad de los sabios más notables de aquella época» (Parcet, 1907 , p. 696), y que «a principios de este siglo recibió en París y Londres singulares muestras de aprecio de parte de sabios de aquellas academias» (Torres Amat, 1836: XXII). Aunque de momento no disponemos de ninguna prueba concreta de su posible encuentro, estas referencias avalarían dicha posibilidad.

Sí que sabemos que el naturalista tarragonés creía haber comprobado experimentalmente que una pequeña y simple criptógama producida por generación espontánea, con el paso de los años podía convertirse en una planta de mayor complejidad. A este respecto Arrau se refería a lo que explicaba Martí en la tertulia en la que participaba en Barcelona, a la que ya nos hemos referido: «... había logrado por este medio plantas acuáticas de un volumen de más de medio pie, con hojas o prolongaciones de la masa, a manera de alas de mariposa (...) En 1829 tenía algunas de estas plantas formadas después de la guerra de la Independencia, como objeto de curiosidad y entretenimiento" (Elias de Molins, 1895, II, p. 94). Es decir que las criptógamas que producía inicialmente se transformaban en plantas acuáticas más grandes y complejas que vivían bastantes años.

Refiriéndose a las técnicas que había desarrollado Martí para producir estos organismos afirmaba su amigo Torres Amat: «... había llegado a saber formarlas, como en efecto las formaba siempre que quería, ya más a prisa, ya más poco a poco, ya grandes, ya pequeñas. Convertía unas criptógamas en otras, formaba fibras vegetales, y de estas pasaba a formar algunas plantas» (Torres Amat, 1836, p. 382)
Es interesante constatar la gran perfección técnica que le atribuye para conseguir producir plantas de muy diversas formas, y como podía transformar unas criptogramas en otras hasta llegar a formar algunas plantas, aumentando por tanto su complejidad.

El mismo Torres Amat recoge en su diccionario una serie de preguntas que Martí hizo en el año 1819 a un clérigo, muy probablemente el mismo, en las que intentaba esclarecer si sus ideas científicas eran compatibles con la fe católica (Torres Amat, 1836, p. 383). Estas preguntas nos permiten conocer algunas de las ideas científicas que Martí ocultaba por miedo a ser considerado hereje. Una de las preguntas fue la siguiente:

¿Y se opone la fe, prosiguió el religioso naturalista, el pensar que la producción de las plantas y animales fue obra de la virtud que dio el Criador a los cuatro elementos, y que esta obra duró muchísimos años?

(Torres Amat, 1836, p. 384).

Esta pregunta pone de manifiesto que Martí pensaba en la posibilidad que Dios hubiese dado a la materia inerte que había creado, la capacidad de ir produciendo las plantas y animales que habitan la Tierra a lo largo de muchísimos años. Estas ideas constituían un primer esbozo de un planteamiento evolucionista deísta.

Quizás el testimonio más importante en relación a esta posibilidad que contemplaba Martí lo encontramos en un texto manuscrito que forma parte del cuadernillo de "Problemas y resultados" en el apartado 10. Consiste en una serie de reflexiones en torno a la producción de las diversas estructuras vegetales a partir de la generación espontánea, donde llega a preguntarse si a partir de este proceso se originarían todas las plantas:

Así como el agua que contiene una mata. [materia] carbonosa miscible con ella, se vuelve amarilla y verde al Sol, que de ella se precipita una mata. verde, que esta se forma a mi voluntad en puntos, faxas, granos, en conclobaciones mas o menos grandes parenchimatosas, en pieles; en substancias gelatinosas, membranáceas, coriáceas, capilares, a saber en plantas vegetantes dentro, o a la superficie de las aguas ¿Podríase también descubrir el mecanismo con que se forman el liber, la madera, las fibras en corticales, como lignosas, las hojas, las flores, etc., mayormente de las plantas que vegetan pte. [preferentemente] en el agua y pte. fuera de ella, de las que necesitan mucha agua, o de la sombra, como musgos, lichenes, etc., en fin dimanarían todas las plantas de aquella agua que observé amarillenta, en que principia la vegetación de una substancia carbonosa destituida un instante antes de lo que se llama vida? (Quintana, 1935, p. 223).

A lo largo de la reflexión admite la posibilidad de que a partir de «materia carbonosa destituida de vida» pudiera formarse "materia verde», organismos fotosintéticos simples, criptógamas, y a partir de ellas 
musgos, líquenes, y plantas superiores con hojas y flores. Es decir admite la posibilidad de evolución de las plantas, que en cierta medida sabemos que creía que había comprobado a través de sus experimentos según los testimonios citados de Parcet y Torres Amat.

Martí parece poder encajar la generación espontánea que originaría los organismos más sencillos en su visión del funcionamiento armónico del conjunto de la naturaleza, en un concepto próximo al equilibrio natural de Lamarck (Corsi, 2001, p. 89). Podemos comprobarlo en otro texto que también procede del manuscrito "Experimentos y observaciones" del 21 de mayo de 1817. Para Martí la generación espontánea sería un medio para la conservación de las especies «...cuando la mano del hombre abusando de los inmensos beneficios del Criador del universo, destruyera alguna de les especies vegetales» (Quintana, 1935, p. 179).

Un elemento necesario para admitir un modelo evolucionista gradualista como el de Lamarck, es la necesidad de enormes períodos de tiempo para producir la gran diversidad de organismos que existen. Martí, que como naturalista también estaba interesado por la geología puesto que había constituido una colección geológica y parece que tenía trabajos inéditos sobre esta materia, defendía que la historia de la Tierra era bastante más larga que la que le atribuía la interpretación literal del Génesis. Los seis días de la creación no corresponderían a períodos de 24 horas sino a seis larguísimos períodos de tiempo, planteamiento que Martí habría explicado a un médico del ejército francés en 1810 (Torres Amat, 1836, p. 384).

La duda de que este planteamiento fuese compatible con la religión católica, también se la planteó al clérigo que visitó en Barcelona, en la entrevista a la que nos hemos referido anteriormente. En la pregunta Martí plantea que la creación de la Tierra podría remontarse a cuarenta mil años o incluso más: «¿Puedo yo sin faltar a la fe sospechar que la creación de cielo y de la tierra, o de los cuatro elementos de que se habla en los primeros versículos del Génesis, hace cuarenta mil años o más que sucedió? ... ¿ंHe de creer como de fe que los seis días de la creación fueros naturales, esto es de 24 horas cada uno?» (Torres Amat, 1836, p. 384).

La idea de una larga historia de la Tierra estaba presente en las Époques de la nature de Buffon, que como hemos visto se encontraba entre las obras de la biblioteca de Martí. También aparece el Système des Animaux sans vertèbres de Lamarck que también poseía Martí, aunque en otros textos del naturalista francés esta idea aparece con más claridad, como por ejemplo en la adición que se incluye en la Philosophie zoologique (Lamarck, 1809, vol. II, p. 474-475).

Una idea importante de los modelos evolucionistas del siglo XIX que no aparece en lo que conocemos de
Martí, es la adaptación de los organismos al medio. El naturalista tarragonés tenía que conocer que Lamarck planteaba como mecanismo fundamental de adaptación al medio la herencia de los caracteres adquiridos. Este planteamiento aparece en el Système des Animaux sans vertèbres, la obra de Lamarck que se conserva de la biblioteca de Martí:

\section{... a causa de estas influencias diversas, las facultades se amplían y se fortifican por el uso, diversificándose por las nuevas costumbres conservadas largo tiempo; y insensiblemente la conformación, la consistencia, en una palabra la naturaleza y el estado de las partes así como de los órganos, participan de las consecuen- cias de todas estas influencias, se conservan y se pro- pagan por la generación (Lamarck, 1801, p. 13).}

Así pues Martí tenía que conocer que Lamarck defendía este mecanismo. Sin embargo, no hemos encontrado en los materiales que se conservan de Martí o en los datos que nos aportan sus contemporáneos, nada que nos permita confirmar que compartía la defensa de la herencia de los caracteres adquiridos como mecanismo de adaptación. Sin embargo, es muy posible que aceptase este tipo de herencia, puesto que la mayor parte de los naturalistas contemporáneos de Martí así lo hacían.

En cambio, sí que mostró interés por descubrir otro mecanismo de cambio de los organismos, la hibridación, por la que también se interesaron muchos evolucionistas del siglo XIX. Al final de la memoria sobre la fecundación de las plantas, Martí manifiesta su aceptación del proceso así como la necesidad de trabajar experimentalmente sobre la hibridación por los beneficios que podría acarrear:

Ya no es permitido el dudar de la hibridéz vegetal que el Señor Koeulreuter ha confirmado igualmente con felices experimentos en las Digitales y Lobebias. Pero es muy de estrañar, atendida la actividad, con que se cultivan algunas partes de la Filosofía natural, que no se haya proseguido un trabaxo tan agradable que, empezado con acierto, parece prometer tantos y tan grandes progresos (Martí, 1791?, p. 80).

También disponemos del testimonio de Parcet, que afirma que Martí consiguió plantas mixtas a partir de la fecundación artificial de especies diferentes (Parcet, 1907, p. 698). Y su amigo, el notable naturalista barcelonés Agustí Yañez, explica que hizo ensayos sobre plantas híbridas desde el último tercio del siglo XVIII, especialmente con cucurbitáceas que reprodujo por espacio de muchos años, haciendo diferentes combinaciones y obteniendo formas extravagantes de melones, pepinos y calabazas (Yañez, 1845: II, 227).

En los años de vida de Martí dos naturalistas publicaron planteamientos evolucionistas más o menos desarrollados: Erasmus Darwin y Lamarck. No tenemos ninguna referencia que nos permita pensar que 
Martí conocía la obra del primero, aunque no lo podemos descartar totalmente. En cambio, Martí conocía el modelo evolucionista de Lamarck, al menos en su primera formulación. Además Martí compartía con el naturalista francés algunas de las ideas básicas de su modelo de evolución: la generación espontánea, la transformación progresiva de los organismos, y un largo tiempo en la historia de la Tierra que permitiera dicha transformación. Pero al no tener constancia de que asumía como mecanismo de adaptación al medio la herencia de los caracteres adquiridos, no podemos afirmar que Martí llegara a asumir el modelo de evolución de Lamarck, aunque sí podemos decir que compartió bastantes de sus ideas.

Estos hechos podrían explicar la influencia de los planteamientos evolucionistas de Lamarck en el citado amigo de Martí, Agustí Yañez. Mucho más joven que el naturalista tarragonés, Yañez le homenajeó en la primera edición de su obra Lecciones de historia natural, al referirse a él como "sabio" y "honor de nuestro principado" (Yañez, 1820, p. 219). Y también en la edición de 1845 , donde refriéndose a la novedad y gran importancia de las investigaciones realizadas por Martí, afirmaba que «si llegasen a publicarse, no dudo que causarán mutaciones de mucha consideración en la fisiología de los vegetales» (Yañez, 1845, vol. II, p. 283).

Esta gran admiración que manifiesta Yañez hacia Martí y su trabajo científico, podría haberle influido en su aceptación de las teorías evolucionistas de Lamarck que puso de manifiesto en el Diccionario pintoresco de Historia Natural y de Agricultura (Sucarrats, 2006, pp. 145-149; Camós, 2007b, pp. 122-129).

\section{CONCLUSIONES}

Como hemos visto, Antoni de Martí i Franquès fue un gran científico que hizo un enorme trabajo experimental en los gabinetes que instaló primero en Altafulla, su pueblo natal, y más tarde en la ciudad de Tarragona. Aunque desarrolló su excepcional trabajo experimental alejado de los lugares donde se producían los grandes avances científicos en Europa, su gran biblioteca, la vinculación a los núcleos científicos barceloneses y su viaje por las principales capitales europeas visitando las instituciones científicas más relevantes, le permitieron conocer con gran prontitud los principales logros científicos que se producían en Europa en el último tercio del siglo XVIII y primeras décadas del siglo XIX.

De su ingente trabajo experimental aquello que tuvo una cierta repercusión tanto en el Estado Español como en los principales centros científicos internacionales, fueron sus ajustados cálculos experimentales sobre la composición del aire atmosférico que fueron publicados en diferentes revistas europeas.
También tuvo repercusión el único trabajo científico que publicó, la memoria sobre la reproducción de los vegetales donde rebatía experimentalmente algunos trabajos del naturalista y sacerdote católico Lazzaro Spallanzani.

Sin embargo conocemos a través del testimonio de sus contemporáneos y de los manuscritos que se conservan de su trabajo experimental, que dedicó un enorme esfuerzo a investigar sobre la generación espontánea de formas vegetales simples que denominaba materia verde, igual que lo hacían otros científicos en diversas partes de Europa que investigaban sobre el mismo tema. También sabemos que Martí estaba convencido de que a través de sus experimentos era capaz de generar formas vivas a partir de materia inanimada, y de desarrollar un cierto control del proceso variando las circunstancias en que lo producía. A partir de los libros que se conservan de su biblioteca, puede comprobarse que recibió influencias sobre este tema de autores como Buffon, Senebier, Priestley y Lamarck. Pero como conocemos que alguno de sus descendientes hizo desaparecer diversos libros sobre la generación espontánea por considerarla contraria a la religión católica, es presumible que Martí también poseyera los libros de los científicos católicos Spallanzani y Needham que desarrollaron un importante debate en torno a este proceso en la segunda parte del siglo XVIII.

A pesar de su enorme trabajo experimental dedicado a la generación espontánea y de su convencimiento de que era capaz de controlar experimentalmente dicho proceso, no publicó ni una sola línea dedicada a este tema. Su profunda religiosidad y el miedo a ser acusado de hereje por buena parte de sus contemporáneos católicos, fue la causa principal de esta autocensura. $Y$ aunque sabemos que su amigo el clérigo liberal Fèlix Torres Amat le explicó que según su criterio sus convicciones científicas no entraban en contradicción con la religión católica, no logró convencer ni a Martí ni a sus herederos de que publicaran los trabajos del naturalista tarragonés dedicados a este delicado y controvertido tema.

Por otra parte también puede constatarse la cercanía del pensamiento de Martí en relación a la aparición y la transformación de los organismos, con el del naturalista Jean Baptiste Lamarck, de quien poseía al menos una obra en su biblioteca, el Système des Animaux sans vertèbres, en la que el naturalista francés desarrolló por escrito una primera formulación de su modelo evolucionista. Además Martí compartía con Lamarck diversos aspectos de su teoría como la generación espontánea de las formas vivas más sencillas, una historia de la Tierra mucho más larga que la que se deducía de la lectura literal del Genesis, así como la posibilidad de que los organismos tendieran 
a transformarse en formas más complejas. Y aunque no sabemos si compartía la defensa de la herencia de los caracteres adquiridos como mecanismo básico en la transformación de los organismos a lo largo del tiempo, sí que conocemos que investigó sobre otro mecanismo de transformación de los organismos, la hibridación.

\section{NOTAS}

1 Este artículo se enmarca en el proyecto "Ciencia y creencia entre dos mundos. Evolucionismo, biopolítica y religión en España y Argentina" (HAR2010-21333-C03-03), financiado por la Dirección General de Investigación, Ministerio de Economía y Competitividad.

2 EXTRACTO "de una memoria sobre los sexos y fecundación de las plantas que el socio libre D. Antonio Martí leyó a la Real Academia Medico Práctica de Barcelona" Diario de los nuevos descubrimientos de todas las ciencias físicas, que tienen alguna relación con las diferentes partes del arte de curar, tomo II, n. V, 321-345. Madrid 1793.

\section{BIBLIOGRAFÍA}

Bernat, Pasqual (2011), “Antoni Martí i Franquès i el sexe de les plantes", Recull de Treballs, 12, pp. 40-51.

Bory de Saint Vincent, J. (1823), "Matière verte". En: Dictionnaire des sciences naturelles, Strasbourg, F. G. Levrault, vol. 29, pp. 324-336.

Buffon (1778), Histoire naturelle générale et particulière: Supplément. Tome cinquième. Des Époques de la nature, París, L'Imprimerie Royale.

Burkhardt, Richard W. (1977), The Spirit of System. Lamarck and Evolucionary Biology, Cambridge, MA, Harvard University Press.

Camós, Agustí (2007a), "Introducció". En: Lamarck, Filosofia zoològica, Col·lecció Clàssics de la Ciència, Barcelona, IEC, ed Pòrtic, Eumo ed.

Camós, Agustí (2007b), De la història natural a l'evolucionisme: Aspectes de l'estudi de la natura a Catalunya i a Espanya als segles XVIII i XIX, Tesis doctoral, dirigida por Jon Arrizabalaga, UAB. http://www.tdx.cat/TDX-0314108-153139.

Caponi, Gustavo (2009), "La miseria de la degeneración: el materialismo de Bufón y las 'limitaciones' de su transformismo", História, Ciências, Saúde- Manguinhos, Rio de Janeiro, 16 (3), pp. 683-703.

Corsi, Pietro (2001), Lamarck. Genèse et enjeux du transformisme 1770-1830, París, CNRS Édit (1a edición en italiano en 1980).

Corsi, Pietro (2012) "Idola Tribus: Lamarck, Politics and Religion in the Early Nineteenth Century". En: Fasolo, Aldo (ed.), The Theory of Evolution and Its Impact, Milan, Springer-Verlag Italia, pp.11-36.

3 SOBRE (1797) "la fecundación de las plantas", Seminario de Agricultura y Artes dirigido a los párrocos, Madrid, 31 de agosto de 1797, p. 120

4 Traducido del catalán, como también haremos de los siguientes textos escritos por Quintana en la misma lengua.

5 Traducido del francés, como también lo haremos en textos posteriores de Buffon, Senebier y Lamarck.

Elias de Molins, Antonio (1895), Diccionario biográfico y bibliográfico de escritores y artistas catalanes del siglo XIX, Barcelona, Imprenta de Calzada, 2 vol.

Farley, John (1977), The spontaneous generation controversy from Descartes to Oparin, Baltimore, Johns Hopkins University Press.

Grapí, Pere (2001), "Antoni Martí i Franquès i la nova química del segle XVII", Estudis Altafullencs, 25, pp. 51-58.

Grau, Josep (2011) “La química de l'aire”. En: Martí, Antoni La química de l'aire, Tarragona, Publicacions URV, pp. 15-46.

Ingen-Housz, Jean (1789), Expériences sur les végétaux, París, Chez Theóphile Barrois.

Lamarck, Jean Baptiste (1801), Système des Animaux sans vertèbres, París, Deterville.

Lamarck, Jean Baptiste (1809), Philosophie Zoologique, París, Dentu, 2 vol.

Martí, Antoni (1791?), Experimentos y observaciones sobre los sexos y fecundación de las plantas, Barcelona, Vda. Piferrer.

Mclaughlin, Peter (2005), "Spontaneous versus Equivocal Generation in Early Modern Science", Annals of the History and Philosophy of Biology, Vol. 10, pp. 79-88.

Mendelsohn, Everett (1976), "Philosophical Biology vs Experimental Biology: Spontaneous Generation in the Seventeenth Century". En: Grene, M.; Mendelsohn, E. (eds.), Topics in the Philosophy of Biology, Boston Studies in the Philosophy of Science 27, Reidel, Dordrecht, pp. 37-65. 
Parcet, Jaume (1907), "Necrología de Dn. Antonio Martí y Franqués escrita por el doctor Jaime Parcet, leída el 20 noviembre 1832", Boletín Arqueológico de Tarragona, 20, 1a. época, pp. 695-700.

Peretó, Juli et alt. (2009), "Charles Darwin and the origin of life", Origins of life Evolution of biospheres, 39, pp. 395-406. [en línea], doi: 10.1007/s11084-009-9172-7.

Quintana Antoni (1935), “Antoni Martí Franquès. Memòries originals. Estudi biogràfic i documental". En: Memòries de l'Acadèmia de Ciències i Arts de Barcelona, tercera época, vol. XXIV.

Quintana, Antoni (1985), “Biografia desapassionada d’Antoni Martí i Franquès". En: Miscel/lania Antoni de Martí i Franquès: amb motiu de la commemoració del 150è aniversari de la seva mort, Tarragona, Ajuntament de Tarragona, pp. 47-88.

Quintana, Antoni (1992), "Epistolari d’Antoni de Martí Franquès...", Estudis Altafullencs, 16, pp. 51-121.

Roe, Shirley A. (1983), "John Turberville Needham and the Generation of Living Organisms", Isis, 74, pp. 159-184.

Roger, Jaques (1993), Les sciences de la vie dans la penseé française au XVIII siècle. La génération des animaux de Descartes à I'Encyclopédie, (1ª edición 1963), Paris, Librairie Armand Colin.
Rovira, Salvador (1982), Antoni Martí Franquès i l'Altafulla del seu temps: 1750-1832, Altafulla, Centre d'Estudis d'Altafulla.

Schofield, R.E. (2004), The Enlightened Joseph Priestley. A Study of His Life and Works from 1773 to 1804, The Pennsylvania State University Press, PA.

Senebier, Jean (1787), “Une ébauche de l'histoire des etres organisés avant leur fécondation". En: Spallanzani, Lazzaro, Éxperiences pour servir a l'histoire de la generation des animaux et des plantes, Geneve, Imprimerie de Bonnat, pp. I-LXXXVIII.

Sucarrats, Raimon (2006), L'ensenyament de la història natural a la Barcelona de la primera meitat del segle XIX. Els llibres de texte i la docència d'Agustí Yañez i Girona, Tesis doctoral, dirigida por Josep Pardo, UAB. http://www.tesisenxarxa.net/TDX-1109106$133436 /$.

Torres Amat, Félix (1836), Memorias para ayudar a formar un diccionario crítico de los escritores catalanes, Barcelona, Imprenta de A. Verdaguer.

Yañez, Agustí (1820), Lecciones de historia natural, Barcelona, Viuda Roca.

Yañez, Agustí (1844/1845), Lecciones de historia natural, Barcelona, Imprenta de Benito Espona y Blai. 